\title{
Estimating Continuous-Time Processes Subject to Time Deformation
}

\author{
An Application to Postwar U.S. GNP
}

\author{
JAMES H. STOCK*
}

\begin{abstract}
A class of time series models is presented in which variables evolve on a data-based rather than calendar time scale. The discrete calendar-time model thus obtained exhibits time-varying parameters and conditional heteroscedasticity. Using a procedure based on the Kalman filter, univariate models are estimated for postwar U.S. real gross national product (GNP) and short- and long-term interest rates. The results indicate significant time scale nonlinearities.
\end{abstract}

KEY WORDS: Nonlinear time series models; Business-cycle analysis; Kalman filter; Unequally spaced data; Time substitution.

\section{INTRODUCTION}

The usual starting point for modeling economic time series is to suppose that the variables can be described by a linear model with time-invariant parameters on a calendar time scale, say quarterly or monthly. But modeling economic time series in calendar time, although convenient, might not capture important nonlinearities if the variables evolve on an operational or economic time scale that differs from calendar time. For example, if time series data on factory output covers a period with a strike, the serial correlation in the series might best be captured using a time scale based on the days during which the plant was operating, rather than simply units of calendar time. More generally, a time scale based on the pace of aggregate economic activity or information flows might be more appropriate for modeling many economic processes than one based on weeks or months. To the extent that economic theory presents no clear guidance on this issue, the question of the appropriate time scale on which to model economic processes is empirical.

This article presents a time series model in which a databased time scale replaces the usual calendar time scale as the basis for the evolution of one or more time series variables. In this "time deformation" model, an $n$-dimensional latent process $\xi(s)$ evolves in "operational" time $s$. The process is observed in calendar time $t$, however, which is related to the operational time scale by a continuous transformation $s=g(t)$. The increments of $g(t)$ are allowed to depend on previously observed variables. The $n$ dimensional calendar time process corresponding to $\xi(s)$ is $Y(t)$; when $Y(t)$ is observed at discrete points in calendar time, it is related to the latent process by $Y(t)=$ $\xi(g(t))$.

The time deformation model has its precursors in the economics and statistics literatures. One important related

* James H. Stock is Assistant Professor of Public Policy, John F. Kennedy School of Government, Harvard University, Cambridge, MA 02138. This research was supported in part by National Science Foundation Grant SES-84-08797. The author is grateful to T. Rothenberg, who supervised the University of California, Berkeley, Ph.D. thesis that this article extends. He also thanks G. Akerlof, C. W. J. Granger, A. Harvey, S. Jones, R. Meese, P. Ruud, M. Stoto, R. Zeckhauser, and the referees for their helpful comments. area concerns the techniques of business-cycle analysis, associated in general with the National Bureau of Economic Research and in particular with Burns and Mitchell (1946). In this work, economic data were transformed to an alternative time scale based on stages of business cycles by "phase averaging," since it was thought that by so doing comovements of macroeconomic variables that were unclear in calendar time would become clear on this "business cycle time" scale. An application of phase averaging appears in Friedman and Schwartz (1982).

This article has four objectives. The first, addressed in Section 2 , is to formulate a multivariate time deformation model in such a way that its unknown parameters can be estimated. The second is to develop an algorithm for estimating the parameters of the model. Such an algorithma maximum likelihood procedure based on the KalmanBucy (1961) filter-is presented in the Appendix. The third objective is to demonstrate that the time deformation model resembles more familiar nonlinear time series models already used successfully with economic data. To this end, Section 3 gives two examples of first order univariate time deformation models. Depending on the form chosen for the time scale transformation, time deformation models are shown to have important similarities to autoregressive conditional heteroscedasticity (ARCH) models and to switching regression models.

The final objective of this article is to use the time deformation model to address three related questions about postwar U.S. GNP. First, is there evidence that GNP evolves on a data-dependent operational time scale? Second, in Stock (1987) I found little evidence in favor of a time scale related to measures of the business cycle using annual U.S. data from 1869 to 1975 . Is there evidence of a business-cycle time scale-as opposed to a more general data-dependent time scale - in the postwar quarterly data set? Third, in calendar time the growth rate of real GNP exhibits weak serial dependence over the postwar period, and the roots of low order estimated discrete-time autoregressive models are real. Is there greater evidence of pseudo-cyclical behavior and higher order dependence,

(c) 1988 American Statistical Association Journal of the American Statistical Association March 1988, Vol. 83, No. 401, Applications 
once the requirement that operational and observational time coincide is relaxed?

I address these three questions in Section 4 by applying the time deformation model to quarterly U.S. economic data from 1954:III to 1983:IV. Estimated univariate time deformation models of the growth rate of real GNP suggest substantial time scale nonlinearities. This inference is supported by estimated bivariate time deformation models, of both GNP growth and short-term interest rates (90-day U.S. Treasury bills) and GNP growth and long-term rates (U.S. Treasury bonds). The estimated time scales are primarily characterized not by cyclical movements, but by an apparent secular acceleration of economic time over the postwar period.

\section{THE TIME DEFORMATION MODEL}

\subsection{The General Model}

The time deformation model relates the latent process $\xi(s)$, evolving in operational time $s$, to the observable process $Y_{t}$, evolving in observational time $t$. When observed at discrete points in calendar time, $Y_{t}$ and the $n$ dimensional latent operational-time process $\xi(s)$ are related by

$$
Y_{t}=\xi(g(t)), \quad t=1,2, \ldots, T,
$$

using the convention that $Y(t)$ refers to the continuous calendar-time process and $Y_{t}$ denotes the corresponding discrete-time variable. Specification of the time deformation model consists of two parts: the choice of $g(t)$ and the specification of the latent process. The latent process is assumed to be generated by a stable continuous (operational) time autoregressive system of order $r$. That is, $\xi(s)$ is assumed to satisfy the linear $r$ th order stochastic differential equation

$$
\begin{aligned}
d\left[D^{r-1} \xi(s)\right]= & {\left[A_{1} D^{r-1} \xi(s)+\cdots+A_{r-1} D \xi(s)\right.} \\
& \left.+A_{r} \xi(s)+X(s ; \beta)\right] d s+d \zeta(s),
\end{aligned}
$$

where $D$ is the mean-square differential operator, $A_{1}, \ldots$, $A_{r}$ are $n \times n$ matrices of coefficients, and $X(s ; \beta)$ is an exogenous variable process that is an integrable function of (operational) time generally depending on an unknown parameter vector $\beta$. The $n$-dimensional process $\zeta(s)$ is assumed to be Gaussian with increments having mean 0 and covariance $E\left[d \zeta(s) d \zeta\left(s^{\prime}\right)^{\prime}\right]=\Sigma d s$ for $s=s^{\prime}$, and equal to 0 for $s \neq s^{\prime}$. [For a precise interpretation of (2.2), see Bergstrom (1983) or Wymer (1972).] It is assumed that $A_{1}, \ldots, A_{r}, \Sigma$, and $\beta$ are known functions of a $k$-dimensional parameter vector $\theta$.

The increments of the time scale transformation are allowed to depend on an $m$ vector of variables $z_{t-1}$ that are observed at or before calendar time $t-1$. Let $\dot{g}(\tau$; $\left.z_{t-1}\right), t-1<\tau \leq t$, denote the time derivative of $g(\tau)$, where the dependence of the increments on $z_{t-1}$ is made explicit; that is, $\dot{g}\left(\tau ; z_{t-1}\right)=d g\left(\tau ; z_{t-1}\right) / d \tau$ for $t-1<$ $\tau \leq t$. In principle, $\dot{\boldsymbol{g}}\left(\tau ; z_{t-1}\right)$ needs to satisfy few restrictions. To facilitate estimation, however, I adopt an exponential parametric form. Letting $c$ be an $m$-dimensional parameter vector, it is assumed that for $t-1<\tau \leq t$,

$$
\dot{g}\left(\tau ; z_{t-1}\right)=\exp \left(c^{\prime} z_{t-1}\right) /\left\{T^{-1} \sum_{t=1}^{T} \exp \left(c^{\prime} z_{t-1}\right)\right\} .
$$

With this specification, if $c=0$ then $\dot{g}\left(\tau ; z_{t-1}\right)=1$, so there are no time deformation effects. Note that $\Delta g(t)=$ $g(t)-g(t-1)=\int_{t-1}^{t} \dot{g}\left(\tau ; z_{t-1}\right) d \tau=\exp \left(c^{\prime} z_{t-1}\right) /\left\{T^{-1}\right.$ $\left.\sum_{t=1}^{T} \exp \left(c^{\prime} z_{t-1}\right)\right\}$. By construction, $T^{-1} \sum_{t=1}^{T} \Delta g(t)=1$ and, as the sample grows large, $E \Delta g(t) \rightarrow 1$. Thus the specification (2.3) has the natural normalization that, on average, operational and observational time progress at the same rate. This identification condition [plus the assumption that $g(0)=0$ ] restricts attention to nonlinearities in the time scale transformation. Furthermore, for finite $c$ and $z_{t-1}, 0<\dot{g}\left(\tau ; z_{t-1}\right)<\infty$, ensuring that operational and observational time proceed in the same direction. Note that although $\dot{g}\left(\tau ; z_{t-1}\right)$ in (2.3) depends on the entire sample of $z$ 's, the observations other than $z_{t-1}$ enter only through the normalizing average in the denominator.

The complete time deformation model is given by (2.2) and (2.3), and is parameterized by the $(k+m)$-dimensional vector $\left(\theta^{\prime} c^{\prime}\right)$.

\subsection{The First Order Case}

The discrete-time representation for multivariate processes obeying (2.2) has been investigated very generally for higher order systems without time deformation [see Bergstrom (1983) and the references therein]. Although the representations for higher order systems are complicated, for first order systems they are not, and it is instructive to examine the time deformation model in this special case. Letting $A_{1}=A$, when $r=1$ and $X(s, \beta)=$ $0,(2.2)$ reduces to $d \xi(s)=A \xi(s) d s+d \zeta(s)$; conditional on $\xi\left(s^{\prime}\right)$, in operational time this has the solution

$$
\xi(s)=e^{A\left(s-s^{\prime}\right)} \xi\left(s^{\prime}\right)+\int_{s^{\prime}}^{s} e^{A(s-r)} d \zeta(r)
$$

for $s>s^{\prime}$, where the matrix exponential is defined by its Taylor series expansion. Thus, letting $s=g(t)$ and $s^{\prime}=$ $g(t-1)$, the observable process has the discrete-time representation

$$
Y_{t}=e^{A \Delta g(t)} Y_{t-1}+v_{t}, \quad v_{t}=\int_{g(t-1)}^{g(t)} e^{A(g(t)-s)} d \zeta(s) .
$$

Evidently time scale shifts induce specific nonlinearities in the discrete calendar-time representation, through both the autoregressive matrix $\exp (A \Delta g(t))$ and the limits of integration of the error term.

\subsection{Estimation}

In economic applications, some of the variables of interest (e.g., the unemployment rate or asset prices) are observed at a specific point in time; that is, they are stock variables. Others (e.g., GNP or investment) are better approximated as integrals over some fixed period of cal- 
endar time, such as a month or quarter; that is, they are flows. The estimation of continuous-time autoregressions using equally spaced data on stock variables and (to a lesser extent) flows has received considerable theoretical attention (see Bergstrom 1976, 1983; Phadke and Wu 1974; A. W. Phillips 1959; P. C. B. Phillips 1972, 1974; Robinson 1976a,b, 1977a; Wymer 1972). The time deformation model, however, has the effect of making the data unequally spaced. Although frequency domain techniques can be used to estimate continuous-time models with irregularly spaced observations for certain classes of time transformations, time domain algorithms are well suited to handling time transformations of the rather general type considered here. [For first order models with specific sampling schemes, see Robinson (1977b) and Stock (1983).] To handle $r$ th order latent processes, the empirical work that follows is performed using an algorithm based on the Kalman filter. A major advantage of the Kalman filter is its direct use of the first order "stacked" form of the $r$ th order system (2.2) to compute the Gaussian likelihood. In first order form, the state vector has a simple transition equation similar to (2.4), though it contains unobserved elements. The Appendix contains an algorithm for estimating higher order mixed stock/flow time deformation models, extending the algorithms of Kalman and Bucy (1961), Jones (1981), and Harvey and Stock (1985).

\section{EXAMPLES}

The time deformation model is related to other previously proposed nonlinear time series models. To clarify these links, in this section two specific time deformation models are presented. Both models are univariate, with a first order latent process and a time transformation of the form (2.3), where $z_{t-1}$ is a scalar random variable; they differ only in the choice of $z_{t-1}$. The first example highlights the similarity between the time deformation model and Engle's (1982) ARCH model. Unlike the ARCH model, however, the parameters of the conditional mean in the time deformation model vary over time through their dependence on the time scale transformation. The second example shows similarities between time deformation and switching regression models. In both examples, letting $X(s$; $\beta)=0$, from (2.4) the discrete-time representation of the observable process is

$$
\begin{aligned}
Y_{t}=e^{A \Delta g(t)} Y_{t-1}+v_{t}, & v_{t} \sim N\left(0, \sigma_{t}^{2}\right), \\
\sigma_{t}^{2} & =-\Sigma\left(1-e^{2 A \Delta g(t)}\right) / 2 A,
\end{aligned}
$$

where the normal distribution is conditional on $\Delta g(t)$ and where $A$ is now a negative scalar.

Example 1: ARCH Models. In this example, let $\dot{g}(\tau$; $z_{t-1}$ ) be given by (2.3) with $z_{t-1}=Y_{t-1}^{2}$. The difference equation (3.1) and this time transformation specify a complete time deformation model. Substituting $\Delta g(t)$ into the autoregressive coefficient and into $\sigma_{t}^{2}$ in (3.1), and expanding the resulting expression for $\sigma_{t}^{2}$ in a Taylor series in the coefficient $c$ appearing in (2.3) around $c=0$, for small time deformations the model has the approximate representation

$$
Y_{t}=\rho_{t-1} Y_{t-1}+v_{t}, \quad \sigma_{t}^{2} \cong \alpha_{0}+\alpha_{1} Y_{t-1}^{2},
$$

where $\rho_{t-1}=\exp \left[A \exp \left(c Y_{t-1}^{2}\right) /\left\{T^{-1} \sum_{t} \exp \left(c Y_{t-1}^{2}\right)\right\}\right], \alpha_{1}=$ $c \Sigma \exp (2 A)$, and $\alpha_{0}=\exp (2 A)\left[(\Sigma / 2 A)-c \Sigma\left(T^{-1} \Sigma_{t}\right.\right.$ $\left.\left.Y_{t-1}^{2}\right)\right]-\Sigma / 2 A$. Note that in large samples $\alpha_{0}$ converges in probability to a constant.

Were it not for the time-varying autoregressive parameter, the model (3.2) would for small-time deformations (i.e., small $c$ ) be a first order ARCH model. The timevarying parameter is, however, a crucial feature implied by the time scale transformation. Furthermore, this variation in the autoregressive parameter is linked to the conditional heteroscedasticity of $v_{t}$ : for positive $c$, when $Y_{t-1}^{2}$ and thus $\Delta g(t)$ are large, $\rho_{t}$ is small (since $A$ is negative by assumption) and $\sigma_{t}^{2}$ is large. That is, when $\Delta g(t)$ is large, a relatively long segment of operational time has progressed during a unit of observational time. In this case the correlation between the observable process and its lag declines, and the variance of the innovation increases. Furthermore, in the form (3.1), $\sigma_{t}^{2}$ will be positive and finite and the system will be nonexplosive as long as a positive and finite amount of operational time has elapsed during a unit of observational time. Using the specification (2.3) this condition will hold as long as $c$ is finite.

Example 2: Switching Regressions. The time deformation model also includes as special cases certain time series switching regressions. Suppose that $\dot{g}\left(\tau, z_{t-1}\right)$ takes on only two values, depending on whether the growth in $Y_{t}$ in the previous period was positive or negative. Letting $z_{t-1}$ equal the sign of $\Delta Y_{t-1}$ and substituting this into (3.1), one finds that the autoregressive coefficient and conditional error variance can each take on two (and only two) values:

$$
\begin{aligned}
Y_{t} & =\gamma_{1} Y_{t-1}+\sigma_{1} u_{t}, & & \Delta Y_{t-1}>0 \\
& =\gamma_{2} Y_{t-1}+\sigma_{2} u_{t}, & & \Delta Y_{t-1} \leq 0,
\end{aligned}
$$

where $u_{t}$ is iid $N(0,1), \gamma_{1}=\exp \left(A c_{1}\right), \gamma_{2}=\exp \left(A c_{2}\right)$, $\sigma_{1}^{2}=-\Sigma\left(1-\exp \left(2 A c_{1}\right)\right) / 2 A$, and $\sigma_{2}^{2}=-\Sigma(1-$ $\left.\exp \left(2 A c_{2}\right)\right) / 2 A$, in which $c_{1}=\exp (c) /\left\{\exp (c) P_{T}+\right.$ $\left.\exp (-c)\left(1-P_{T}\right)\right\}$ and $c_{2}=c_{1} \exp (-2 c)$, where $P_{T}=$ $T^{-1} \Sigma_{t} 1\left(\Delta Y_{t-1}>0\right)$. As $T$ increases, $P_{T} \stackrel{p}{\rightarrow} P\left(\Delta Y_{t-1}>\right.$ $0)$. The discrete (calendar) time process (3.3) thus switches between two regimes, depending on whether the growth in the previous period was positive or negative. This switching model can be extended in several ways. For example, the switching criterion could be based on growth over several previous periods-as is done in the empirical work that follows-or the switching criterion itself could involve unknown parameters. Alternatively, several regimes could be included rather than just two.

The switching model (3.3) has two distinctive features. First, the variances of the errors in the two regressions differ. Second, the time deformation model restricts the values that $\gamma_{1}$ and $\gamma_{2}$ can attain: Since $\ln \left(\gamma_{1} / \gamma_{2}\right)=$ $A(\exp (2 c)-1) /\left\{1+(\exp (2 c)-1) P_{T}\right\}$, if (for example) $P_{T}=.5$, then as long as $c$ is finite $\left|\ln \left(\gamma_{1} / \gamma_{2}\right)\right|<-2 A$. These features arise because the nonlinearities in (3.3) 
stem from the same source, the time scale transformation. And though the unrestricted switching model has four free parameters $\left(\gamma_{1}, \gamma_{2}, \sigma_{1}^{2}\right.$, and $\left.\sigma_{2}^{2}\right)$, the restricted model (3.3) has only one, $c$.

\section{AN ANALYSIS OF POSTWAR U.S. GNP}

In this section, the Kalman filter algorithm presented in the Appendix is used to investigate whether postwar U.S. GNP is subject to time deformation. I approach the three questions raised in the introduction, concerning the cyclical nature of time deformation in GNP, by estimating three sets of models: (a) univariate models of real GNP, (b) bivariate models of GNP and the 90-day U.S. Treasury bill rate, and (c) bivariate models of GNP and the yield on long-term U.S. Treasury bonds. The estimates are based on quarterly data from 1954:III to 1983:IV, with earlier observations used for initial values as necessary.

\subsection{Univariate Real GNP}

I start by summarizing the discrete-time properties of real GNP as reflected in low order univariate time series models. Interpreted narrowly, business cycles can be thought of as implying a spectral peak at business-cycle frequencies (periods of approximately 2-6 years). (A multivariate characterization of business cycles would emphasize spectral power and high coherence across several time series at low frequencies. An analysis of these multivariate properties in operational time is, however, outside the scope of this investigation.) It has been observed (see, for example, Nelson and Plosser 1982) that, using conventional modeling techniques, GNP growth appears to be well fit by a univariate process with real roots, specifically by first order autoregressive (AR) or moving average (MA) models. Although Harvey (1985) suggested that this result is sensitive to the estimation period-in particular, spectral peaks indicating business-cycle behavior are more evident in the prewar than the postwar period-the absence of complex roots in GNP contrasts with the view that economic activity fluctuates cyclically. This lack of pseudocyclical behavior in the postwar data is evident in the AR(1) and AR(2) discrete-time models fit to $y_{t}=100^{*} \ln \left(I_{t} / I_{t-1}\right)$, where $I_{t}$ is seasonally adjusted real GNP; the results are summarized as Models 1 and 2 in Table 1 . The second autoregressive coefficient in the discrete-time AR(2) model is small and differs insignificantly from 0 , with both models having Box-Pierce $Q(20)$ statistics equaling 13.6, indicating a lack of serial correlation in the residuals. In addition, the roots of the $\operatorname{AR}(2)$ models are both real.

Part B of Table 1 contains estimated continuous-time models of GNP growth without time deformation, where $y_{t}$ is treated as a flow variable (the integral of the instantaneous growth rate from $t-1$ to $t$ ). The results are similar to those for the discrete-time models. In particular, the continuous time AR(2) (Model 4) exhibits extremely large $\mathrm{AR}$ coefficients and a root of $-6,540$, indicative of overfitting the continuous-time AR model. Since these models exhibit only one nontrivial real root, this evidence suggests a lack of a stochastic cycle in quarterly GNP growth.

Are these results to be interpreted as evidence against the existence of business cycles in real GNP? Not necessarily, if the cyclical behavior occurs in economic rather than in calendar time. To investigate this possibility, two time deformation models were fit to $y_{t}$, in which the latent process was assumed to evolve according to an $\operatorname{AR}(2)$ in continuous operational time. The time scale transformations are of the form (2.3), with $z_{t-1}$ containing lagged values of both $y_{t}$ and a cyclical indicator variable, $B_{t}$. This indicator equals +1 during growth expansions and -1 during growth contractions; it switches if (a) the growth in each of the previous two quarters was greater (less) than the postwar average but (b) $B_{t-1}$ still indicates a contraction (expansion). Thus $B_{t}$ depends only on values of GNP observed by time $t$, avoiding the difficulties associated with forward-looking cyclical indicators. [For further

Table 1. Univariate Models of Real GNP Growth, 1954:III-1983:IV

\begin{tabular}{|c|c|c|c|c|c|c|c|}
\hline \multirow[b]{2}{*}{ Model } & \multicolumn{4}{|c|}{$z_{t-1}$} & \multirow[b]{2}{*}{ Roots } & \multirow{2}{*}{$\begin{array}{l}\text { Log- } \\
\text { likelihood }\end{array}$} & \multirow[b]{2}{*}{ MSE } \\
\hline & $y_{t-1}$ & $y_{t-2}$ & $B_{t-1}$ & $B_{t-2}$ & & & \\
\hline \multicolumn{8}{|c|}{ A. Discrete time } \\
\hline 1. $\operatorname{AR}(1): y_{t}=\underset{(.110)}{.504}+. .377 y_{t-1}$ & - & - & - & - & .377 & - & .922 \\
\hline $\begin{aligned} & \text { 2. } \operatorname{AR}(2): y= \\
&.502+.118)(.092) \\
&\left(.09 y_{t-1}\right.+.005 y_{t-2} \\
&(.091)\end{aligned}$ & - & - & - & - & $(.39,-.01)$ & - & .922 \\
\hline \multicolumn{8}{|c|}{ B. Continuous time (flows) } \\
\hline 3. $A R(1): D y(t)=1.523-1.882 y(t)$ & - & - & - & - & -1.88 & -163.47 & .942 \\
\hline 4. AR(2): $D^{2} y(t)=9,957-6,542 D y(t)-12,310 y(t)$ & - & - & - & - & $(-1.88,-6,540)$ & -163.47 & .942 \\
\hline \multicolumn{8}{|c|}{ C. Continuous time/Time deformation } \\
\hline 5. $\operatorname{AR}(2): D^{2} \xi(s)=29.6-8.80 D \xi(s)-38.3 \xi(s)$ & $\begin{array}{l}.043^{\mathrm{a}} \\
(.014)\end{array}$ & $\begin{array}{l}.228^{\mathrm{a}} \\
(.057)\end{array}$ & - & - & $(-4.40 \pm 4.35 i)$ & -148.47 & .906 \\
\hline 6. $\operatorname{AR}(2): D^{2} \xi(s)=29.2-9.15 D \xi(s)-36.9 \xi(s)$ & $\begin{array}{l}.012^{b} \\
(.007)\end{array}$ & $\begin{array}{l}.158^{\mathrm{a}} \\
(.034)\end{array}$ & $\begin{array}{l}.102 \\
(.166)\end{array}$ & $\begin{array}{l}.425 \\
(.620)\end{array}$ & $(-4.58 \pm 4.00 i)$ & -147.81 & .901 \\
\hline
\end{tabular}

NOTE: The estimated time scale transformations are of the form (2.3). Numerical standard errors are given in parentheses.

a Differs significantly from 0 at the $1 \%$ level.

b Differs significantly from 0 at the $10 \%$ level. 
discussion of the problem of identifying business-cycle turning points, see Neftci $(1984,1985)$.]

Two estimated time deformation models are presented as Models 5 and 6 in Table 1 . In Model $5, z_{t-1}$ consists of two variables, the first and second lags of real GNP. Both coefficients in the estimated transformation are positive and significant at the 5\% level. According to the estimated parameters, a sustained increase in the GNP growth rate by .01 percentage points for two consecutive quarters is associated with an increase in the rate of evolution of economic time by $.27 \%$. Model 6 examines a time scale transformation that incorporates both lagged values of the growth rate of real GNP and two lags of the business-cycle variable $B_{t}$. The marginal effect of these business-cycle variables is small: The hypothesis that the coefficients on $B_{t-1}$ and $B_{t-2}$ equal 0 cannot be rejected at the $10 \%$ level. In both models, there is a small but noticeable improvement in the in-sample one-step-ahead forecasts, with mean squared errors (MSE's) dropping by $5 \%$. These results point to a time scale based on lagged values of GNP itself rather than on the cyclical indicator.

In contrast to the models without time deformation, in both Models 5 and 6 the latent processes exhibit pseudocyclical behavior in operational time as indicated by the complex roots of the estimated AR(2). The cyclical periods corresponding to these roots are 1.4 and 1.6 units of operational time for Models 5 and 6, respectively. Since one unit of operational time has been normalized to equal one unit of calendar time on average, this corresponds to an average period in calendar time of $1 \frac{1}{2}$ quarters for this stochastic cycle. Conclusions drawn from these calculations must be tempered by the lack of confidence intervals associated with these estimated periods. But the point estimates suggest that though GNP growth exhibits stochastic cyclical behavior in operational time, the associated periodicity is much shorter than would be expected for the business cycle.

\subsection{Real GNP and Interest Rates}

I now turn to bivariate time deformation models with $y_{t}$ and two interest rates. The first set of models examines GNP growth and a short-term interest rate, the 90-day U.S. Treasury bill rate available on the last day of the quarter; the second set examines GNP growth and the yield on a portfolio of U.S. Treasury bonds with maturities in excess of 10 years. In the bivariate continuous-time models, GNP growth is treated as a flow, and the interest rates are modeled as stock variables.

Turning first to the GNP/Treasury bill analysis, a bivariate first order discrete-time vector autoregression and its continuous-time counterpart appear in Part A of Figure 1. Diagnostics such as the MSE and the Box-Pierce statistic indicate that these two models fit the data approximately equally well, with the MSE for the discrete-time model being slightly less than for the continuous-time model. Four bivariate time deformation models involving a first order autoregressive latent process and different time scale transformations are presented in Part A of Table 2. Based on the likelihood ratio statistics, all models indicate significant time deformation effects at the $5 \%$ level,

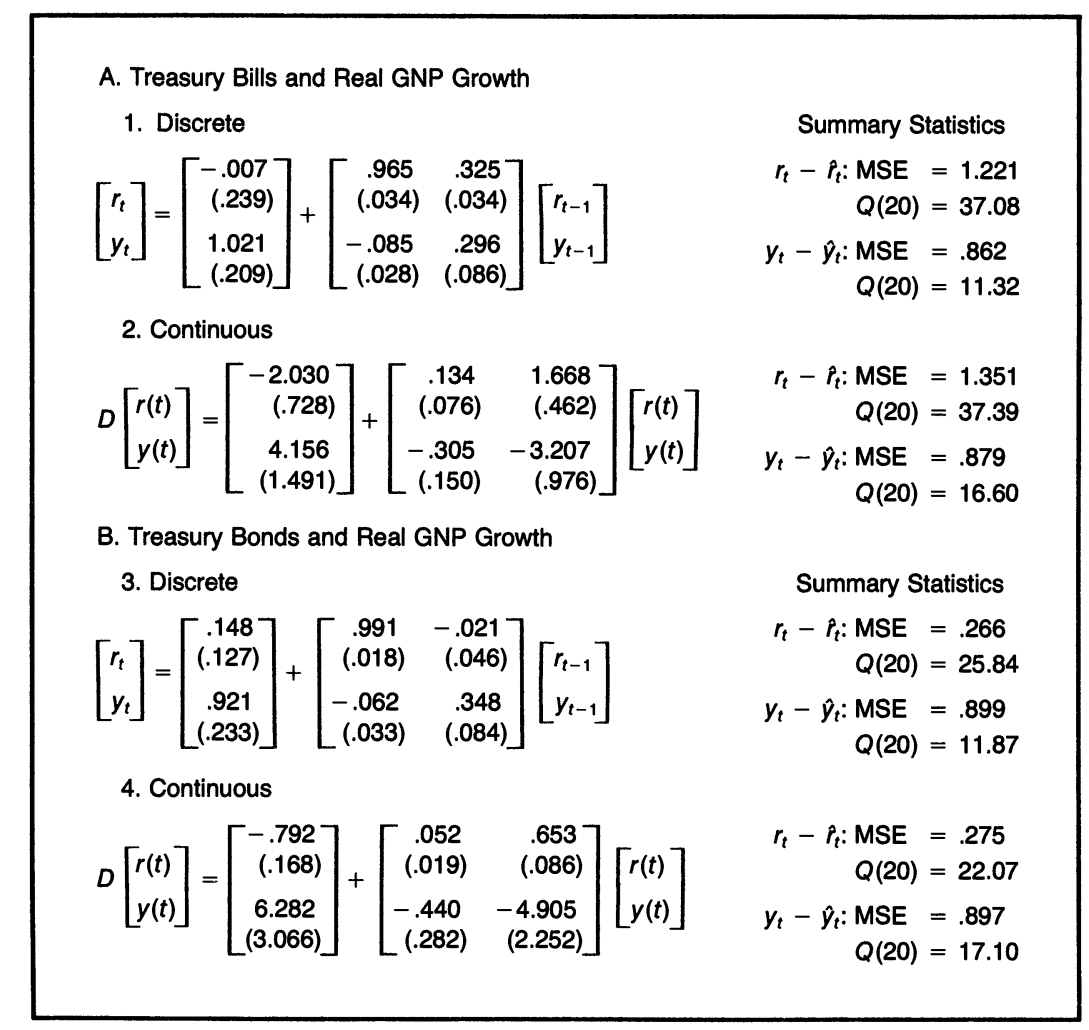

Figure 1. Bivariate Discrete- and Continuous-Time First Order Models Without Time Deformation, 1954:III-1983:IV. Numerical standard errors are given in parentheses. Q(20) denotes the Box-Pierce $Q$ statistic with 20 degrees of freedom. 
Table 2. Estimated Time Scale Transformations for First Order Bivariate Systems With Real GNP Growth and Interest Rates, 1954:III-1983:IV

\begin{tabular}{|c|c|c|c|c|c|c|c|c|c|c|c|}
\hline \multirow[b]{2}{*}{ Model } & \multicolumn{6}{|c|}{$z_{t-1}$} & \multicolumn{2}{|c|}{$Q(20)$} & \multicolumn{2}{|c|}{ MSE } & \multirow[b]{2}{*}{$L R$} \\
\hline & $r_{t-1}$ & $r_{t-2}$ & $y_{t-1}$ & $y_{t-2}$ & $B_{t-1}$ & $B_{t-2}$ & $r_{t}-\hat{r}_{t}$ & $y_{t}-\hat{y}_{t}$ & $r_{t}-P_{t}$ & $y_{t}-\hat{y}_{t}$ & \\
\hline \multicolumn{12}{|c|}{ A. Treasury bills and real GNP growth } \\
\hline 1 & $\begin{array}{r}.040^{\mathrm{a}} \\
(.012)\end{array}$ & - & $\begin{array}{l}.047^{\mathrm{a}} \\
(.014)\end{array}$ & - & - & - & 19.33 & 16.76 & 1.445 & .874 & $14.17^{\mathrm{a}}$ \\
\hline 2 & $\begin{array}{l}.040^{\mathrm{a}} \\
(.013)\end{array}$ & $\begin{array}{c}.000 \\
(.001)\end{array}$ & $\begin{array}{l}.047^{\mathrm{a}} \\
(.015)\end{array}$ & $\begin{array}{l}.000 \\
(.001)\end{array}$ & - & - & 19.33 & 16.76 & 1.445 & .878 & $14.21^{\mathrm{a}}$ \\
\hline 3 & - & - & - & - & $\begin{array}{r}-.024^{b} \\
(.011)\end{array}$ & $\begin{array}{r}.010^{b} \\
(.005)\end{array}$ & 24.95 & 16.14 & 1.431 & .884 & $7.72^{b}$ \\
\hline 4 & $\begin{array}{r}.040^{\mathrm{a}} \\
(.014)\end{array}$ & $\begin{array}{c}.001 \\
(.001)\end{array}$ & $\begin{array}{c}.047^{\mathrm{a}} \\
(.017)\end{array}$ & $\begin{array}{l}.000 \\
(.001)\end{array}$ & $\begin{array}{c}.000 \\
(.001)\end{array}$ & $\begin{array}{c}.000 \\
(.001)\end{array}$ & 19.33 & 16.77 & 1.388 & .886 & $14.67^{\mathrm{b}}$ \\
\hline \multicolumn{12}{|c|}{ B. Treasury bonds and real GNP growth } \\
\hline 5 & $\begin{array}{c}.090^{\mathrm{a}} \\
(.011)\end{array}$ & - & $\begin{array}{l}.014^{\mathrm{a}} \\
(.004)\end{array}$ & - & - & - & 24.51 & 14.90 & .272 & .948 & $34.12^{\mathrm{a}}$ \\
\hline 6 & $\begin{array}{l}.062^{\mathrm{a}} \\
(.011)\end{array}$ & $\begin{array}{r}-.021^{\circ} \\
(.011)\end{array}$ & $\begin{array}{c}-.002 \\
(.004)\end{array}$ & $\begin{array}{l}.018^{\mathrm{a}} \\
(.003)\end{array}$ & - & - & 21.82 & 17.70 & .276 & .861 & $34.60^{\mathrm{a}}$ \\
\hline 7 & - & - & - & - & $\begin{array}{r}-.010^{\mathrm{a}} \\
(.002)\end{array}$ & $\begin{array}{l}.003^{\mathrm{a}} \\
(.001)\end{array}$ & 24.06 & 15.22 & .269 & .967 & $16.60^{\mathrm{a}}$ \\
\hline 8 & $\begin{array}{l}.062^{a} \\
(.011)\end{array}$ & $\begin{array}{r}-.021^{\circ} \\
(.012)\end{array}$ & $\begin{array}{c}-.002 \\
(.004)\end{array}$ & $\begin{array}{l}.018^{\mathrm{a}} \\
(.003)\end{array}$ & $\begin{array}{c}-.003 \\
(.002)\end{array}$ & $\begin{array}{l}.002 \\
(.002)\end{array}$ & 21.88 & 14.63 & .276 & .838 & $38.32^{\mathrm{a}}$ \\
\hline
\end{tabular}

NOTE: Numerical standard errors are given in parentheses. The likelihood ratio statistic tests the hypothesis that $c=0$, and is asymptotically distributed as $\chi_{m}^{2}$, where $m$ is the dimension of $c$.

a Differs significantly from 0 at the $1 \%$ level.

${ }^{b}$ Differs significantly from 0 at the $5 \%$ level.

c Differs significantly from 0 at the $10 \%$ level.

and two do so at the $1 \%$ level. The expansion/contraction switching variable is significant when it appears alone in the time scale transformation. When lagged interest rates and GNP are added, however, the additional explanatory contribution of the cyclical variable is negligible: The likelihood ratio statistic testing the hypothesis that the coefficients on $B_{t-1}$ and $B_{t-2}$ in Model 4 of Table 2 are 0 (distributed $\chi_{2}^{2}$ ) equals .46 . Thus as for the univariate models in Table 1, the marginal contribution of the switching variables to the estimated operational time scale for this bivariate process appears to be small.

Estimated bivariate models of GNP growth and Treasury bond yields are reported in Part B of Table 2 and Figure 1 . The results are broadly similar to those for Treasury bills. The discrete- and continuous-time models fit the data approximately equally well. Comparing the estimated time deformation models in Parts A and B of Table 2, the time deformation effects with bonds are even more striking than with bills: The likelihood ratio statistics are all greater for bonds, as are the estimated coefficients of the time scale transformations. As in Model 4, the marginal contribution of the switching variables in Model 8 is not statistically significant at the $10 \%$ level, although by themselves the switching variables enter the time transformation significantly at the $1 \%$ level.

The estimated operational time scale corresponding to Model 4 in Table 2 is plotted in Figure 2, with the National Bureau of Economic Research business-cycle recessions marked by shaded areas. The correlation between this scale and the corresponding time scale with Treasury bonds

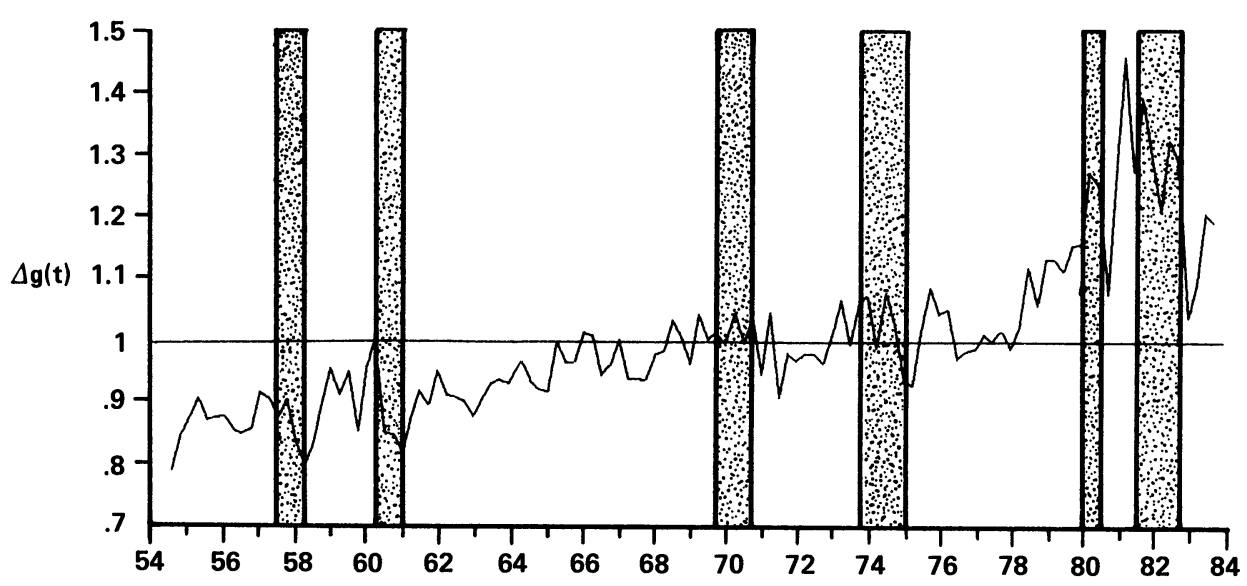

Figure 2. Estimated Time Scale Transformation for GNP and the 90-Day Treasury Bill Rate. The shaded areas denote NBER-dated recessions. The time transformation $\Delta g(t)$ is the estimate obtained from Model 4 of Table 2. 
(Model 8 in Table 2) is high, .91, although this might not be surprising, since long rates and short rates are themselves highly correlated. Because a multivariate model with different time scales for each series has not been defined, I do not formally test the hypothesis that these two time transformations are the same. They do, however, have many common features. For example, according to both estimated transformations, operational time has been progressing increasingly more rapidly than observational time over the past 30 years: the pace of economic activity has quickened. In addition, both scales provide scant evidence in favor of a time scale based largely on the business cycle: Although the GNP/Treasury bill time scale declines during four of the cyclical contractions (Fig. 2), the correlation between the estimated time scales and the contraction/ expansion indicator $B_{t-1}$ is -.05 for the GNP/Treasury bill scale and -.08 for the GNP/Treasury bond scale. Clearly, this indicator accounts for only a small part of the overall variation in the time scales. This conclusion is consistent with the results in Stock (1987) mentioned in the introduction.

The secular trend in $\Delta g(t)$ in Figure 2 implies changing serial correlations in GNP growth and interest rates. For example, when one uses the univariate formulation (3.1) and the estimated operational-time AR parameter from Model 3 of Table 1, the discrete calendar-time first order AR coefficient for GNP growth ranges from $\exp (-1.88 \times$ $.8)=.22$ at the beginning of the sample to .06 during the early 1980s. Thus for most of the sample, GNP growth exhibits modest first order dependence in calendar time. During the turbulent late 1970s and early 1980s, however, its calendar time representation appears closer to a random walk, with an increased conditional error variance.

\section{CONCLUSIONS}

Time deformation models can be estimated successfully using the Kalman filter algorithm presented in the Appendix. This algorithm makes possible joint estimation of the parameters describing the evolution of the latent process in operational time and the parameters of the time scale transformation. In response to the questions about postwar GNP raised in the introduction, there is evidence that GNP evolves on a time scale other than calendar time. Lags of both GNP growth itself and interest rates are seen to be statistically significant determinants of this time scale. The estimated time deformation models also suggest that GNP exhibits stochastic cyclical behavior in operational time rather than in observational time, although the periodicity of this cycle is much shorter than the periods typically associated with business cycles. Although the empirical importance of time deformation in describing variables other than GNP remains to be seen, these initial results suggest that the time deformation model might be appropriate for a variety of macroeconomic variables.

\section{APPENDIX: THE KALMAN FILTER FOR TIME DEFORMATION MODELS}

This appendix presents the equations of the Kalman filter algorithm for estimating the parameters of a higher order mixed stock/flow continuous-time system, subject to time deformation, and sketches their derivation. In general, there will be $n_{s}$ stock variables $Y_{t}^{s}$ and $n_{f}$ flow variables $Y_{t}^{f}$. Ordering $Y_{t}$ with the stock variables first and allowing for the possibility that $Y_{t}$ might be observed with error, the observable process is given by

$$
Y_{t}=\left[\begin{array}{c}
Y_{t}^{s} \\
Y_{t}^{f}
\end{array}\right]=\left[\begin{array}{c}
\xi(g(t)) \\
\int_{t-1}^{t} \xi(g(r)) d r
\end{array}\right]+\varepsilon_{t}, \quad t=1,2, \ldots, T,
$$

where $\varepsilon_{t}=\left(\varepsilon_{t}^{s \prime} \varepsilon_{t}^{f \prime}\right)^{\prime}$ is an $n=\left(n_{s}+n_{f}\right)$-dimensional white noise measurement error independent of $\zeta(s)$, with $E \varepsilon_{t}=0$ and $E \varepsilon_{t} \varepsilon_{t}^{\prime}=H$.

The Kalman filter is used to evaluate the Gaussian likelihood function in the time domain, given a trial parameter vector. The filter employs a discrete-time state space formulation implied by (2.2) and (2.3), with certain elements of the state vector observable as $Y_{t}$ (perhaps plus the measurement error $\varepsilon_{t}$ ). This appendix extends the Kalman-Bucy (1961) filter in two ways. First, some of the variables are observed as flows, and their transition equations and Kalman gains are treated separately from those for stocks. Second, the time deformation model imparts nonlinearities to the process, resulting in time-varying filter parameters that depend on $\Delta g(t)$.

Estimation proceeds by numerical maximization of the Gaussian likelihood over the elements of $\theta$. [For a discussion of the identifiability of these parameters and related references see Stock (1987).]

State Transition Equation. In operational time, the stochastic differential equation (2.2) can be written in stacked form as

$$
d \Psi^{*}(s)=\left[A \Psi^{*}(s)+R X(s ; \beta)\right] d s+R d \zeta(s),
$$

where

$$
\Psi^{*}(s)=\left[\begin{array}{c}
\xi(s) \\
D \xi(s) \\
\vdots \\
D^{r-l} \xi(s)
\end{array}\right], \quad R=\left[\begin{array}{c}
0 \\
\vdots \\
I
\end{array}\right], \quad A=\left[\begin{array}{cc}
0 & \\
\vdots & I \\
0 & \\
A_{r} & A_{r-l} \cdots A_{1}
\end{array}\right]
$$

The roots of $A$ have negative real parts by the assumption of stability. Following Jones (1981) and Harvey and Stock (1985), it is convenient to assume that the roots are distinct and to adopt the eigenvalue decomposition $A=G \Lambda G^{-1}$, where $\Lambda$ is a matrix with the (generally complex) eignevalues of $A$ on the diagonal and zeros off the diagonal, and where the rows of $G$ contain the eigenvectors of $A$. Setting $\Psi(s)=G^{-1} \Psi^{*}(s)$, (A.1) becomes $d \Psi(s)=\left[\Lambda \Psi(s)+G^{-1} R X(s ; \beta)\right] d s+G^{-1} R d \zeta(s)$. Thus for $s>s^{\prime}, \Psi(s)$ satisfies

$\Psi(s)=e^{\Lambda\left(s-s^{\prime}\right)} \Psi\left(s^{\prime}\right)$

$$
+\int_{r=s^{\prime}}^{s} e^{\Lambda(s-r)} G^{-1} R X(r ; \beta) d r+\int_{r=s^{\prime}}^{s} e^{\Lambda(s-r)} G^{-1} R d \zeta(r) .
$$

Let the calendar-time state vector be $\alpha(\tau)=\Psi(g(\tau))$, and let $s=g(\tau)$ and $s^{\prime}=g(t-1)$. From (A.2), $\alpha(\tau)$ evolves according to

$$
\begin{aligned}
\alpha(\tau)=e^{\Lambda(g(\tau)-g(t-1))} \alpha(t-1) & +\int_{r=g(t-1)}^{g(\tau)} e^{\Lambda(g(\tau)-r)} G^{-1} R X(r ; \beta) d r \\
& +\int_{r=g(t-1)}^{g(\tau)} e^{\Lambda(g(\tau)-r)} G^{-1} R d \zeta(r) .
\end{aligned}
$$

To handle flow variables, let $\alpha_{t}^{f}$ be the integral of $\alpha(\tau)$ over the calendar-time period from $t-1$ to $t$; that is, $\alpha_{t}^{f} \equiv \int_{t-1}^{t} \alpha(\tau)$ $d \tau$, where $\alpha_{t}$ denotes $\alpha(t)$ at discrete points in calendar time. The evolution of this $n r \times 1$ vector, obtained by integrating $\alpha(\tau)$ 
in (A.3) from $t-1$ to $t$, is given by

$$
\alpha_{t}^{f}=W_{t} \alpha_{t-1}+\gamma_{t}^{f}+v_{t}^{f}
$$

where $W_{t}=\int_{t-1}^{t} \exp \{\Lambda(g(\tau)-g(t-1))\} d \tau$ and where

$$
\gamma_{t}^{f}=\int_{\tau=t-1}^{t} \int_{r=g(t-1)}^{g(\tau)} e^{\Lambda(g(\tau)-r)} G^{-1} R X(r ; \beta) d r d \tau
$$

and

$$
v_{t}^{f}=\int_{\tau=t-1}^{t} \int_{r=g(t-1)}^{g(\tau)} e^{\Lambda(g(\tau)-r)} G^{-1} R d \zeta(r) d \tau .
$$

Using (A.3) evaluated at $\tau=t$ and (A.4), the evolution of the state vectors of the combined stock/flow system in calendar time is summarized by

$$
\left[\begin{array}{l}
\alpha_{t} \\
\alpha_{t}^{f}
\end{array}\right]=\left[\begin{array}{ll}
T_{t} & 0 \\
W_{t} & 0
\end{array}\right]\left[\begin{array}{l}
\alpha_{t-1} \\
\alpha_{t-1}^{f}
\end{array}\right]+\left[\begin{array}{l}
\gamma_{t} \\
\gamma_{t}^{f}
\end{array}\right]+\left[\begin{array}{l}
v_{t} \\
v_{t}^{f}
\end{array}\right],
$$

where $T_{t}=\exp (\Lambda \Delta g(t)), \gamma_{t}=\int_{g(t-1)}^{g(t)} \exp \{\Lambda(g(t)-r)\} G^{-1} R X(r$ $\beta) d r$, and $v_{t}=\int_{g(t-1)}^{g(t)} \exp \{\Lambda(g(t)-r)\} G^{-1} R d \xi(r)$. Letting $\alpha_{t}^{\dagger}=$ $\left(\alpha_{t}^{\prime} \alpha_{t}^{f}\right)^{\prime}$ and adopting obvious simplifying notation, (A.5) becomes

$$
\alpha_{t}^{\dagger}=T_{t}^{\dagger} \alpha_{t-1}^{\dagger}+\gamma_{t}^{\dagger}+v_{t}^{\dagger} .
$$

Measurement Equation, the Kalman Filter, and Initial Conditions. Let $I_{k}$ denote the $n_{k} \times n_{k}$ identity matrix. The measurement equations now are

$$
Y_{t}^{s}=\left[\begin{array}{llll}
I_{n_{s}} & \cdots & 0
\end{array}\right] G \alpha_{t}+\varepsilon_{t}^{s}
$$

and

$$
Y_{t}^{f}=\left[\begin{array}{lllll}
0 & I_{n_{f}} & \cdots & 0
\end{array}\right] G \alpha_{t}^{f}+\varepsilon_{t}^{f},
$$

where the first block of zeros in (A.7b) has dimension $n_{f} \times n_{s}$.

The equations of the Kalman filter with complex state variables now follow directly from the transition equation (A.6) for the augmented state vector and from the measurement equations (A.7) (see Harvey and Stock 1985; Jones 1981; Kalman and Bucy 1961).

Initial conditions for the augmented state vector $\alpha_{t}^{\dagger}$ and the covariance matrix of the one-step-ahead forecast of the state vector $P_{t \mid t-1}^{\dagger}$ can be obtained by calculating the unconditional mean and covariance matrix of the state vector. Since the time scale transformation depends on $z_{t-1}$, these unconditional expectations are not readily evaluated. Instead, the initial conditions are derived assuming that prior to the observation period, $\Delta g(t)=1$. In this case, $a_{1 \mid 0}^{\dagger}$ and $P_{1 \mid 0}^{\dagger}$ can be obtained by iterating the state transition equation backwards and taking unconditional expectations. Thus $a_{1 \mid 0}^{\dagger}=\sum_{j=0}^{\infty}\left(T^{\dagger}\right)^{j+1} \gamma_{1-j}$ and $P_{1 \mid 0}^{\dagger}=$ $\sum_{j=0}^{\infty}\left(T^{\dagger}\right)^{j} Q\left(\bar{T}^{\dagger^{\prime}}\right)^{j}$, where $T^{\dagger}$ and $Q$ denote $T_{t}^{\dagger}$ and $Q_{t}$ evaluated with $\Delta g(t)=1$ and where $\bar{T}^{\dagger}$ denotes the complex conjugate of $T^{\dagger}$.

Integrals Comprising the Covariance Matrix and Deterministic Terms. The nonstochastic integrals entering (A.6) and the filter gain (through the error covariance matrix) will in general depend on $g(t)$ for all $t$. For convenience, in the empirical work reported in Section 4 it is assumed that $g(t)$ is piecewise linear; that is, for $t-1<\tau \leq t, g(\tau)=g(t-1)+(\tau-(t-1)) \Delta g(t)$, where $\Delta g(t)$ is given by (2.3). An alternative would be to interpolate $g(t)$ using a more sophisticated smoothing algorithm, although such a strategy is not pursued here. Let

$$
Q_{t}=E v_{t}^{\dagger} \bar{v}_{t}^{\prime \prime}=\left[\begin{array}{ll}
Q_{t}^{s s} & Q_{t}^{s f} \\
Q_{t}^{s f} & Q_{t}^{f f}
\end{array}\right]
$$

Also let $q_{i j} \equiv\left(G^{-1} R \Sigma R^{\prime} \bar{G}^{-1 \prime}\right)_{i j}$ and let $\lambda_{i}, T_{i t}$, and $W_{i t}$ denote the $(i, i)$ element of the diagonal $n r \times n r$ matrices $\Lambda, T_{t}$, and $W_{t}$, respectively. Assuming piecewise linearity of $g(t)$, the $(i, j)$ elements of the constituent blocks of the conditional error co- variance matrix $Q_{t}$ are given by

$$
\begin{aligned}
\left(Q_{t}^{s s}\right)_{i j}= & q_{i j} \int_{r=0}^{\Delta g(t)} \exp \left\{\left(\lambda_{i}+\bar{\lambda}_{j}\right)(\Delta g(t)-r)\right\} d r \\
= & -q_{i j}\left(1-T_{i t} \bar{T}_{j t}\right) /\left(\lambda_{i}+\bar{\lambda}_{j}\right), \\
\left(Q_{t}^{s f}\right)_{i j}= & {\left[q_{i j} /\left(\bar{\lambda}_{j} \Delta g(t)\right)\right] \int_{r=0}^{\Delta g(t)} \exp \left\{\lambda_{i}(\Delta g(t)-r)\right\} } \\
& \times\left(\exp \left\{\bar{\lambda}_{j}(\Delta g(t)-r)\right\}-1\right) d r \\
= & \left\{\left(Q_{t}^{s s}\right)_{i j}-W_{i t} q_{i j} \Delta g(t)\right\} /\left(\bar{\lambda}_{j} \Delta g(t)\right),
\end{aligned}
$$

and

$$
\begin{aligned}
\left(Q_{t}^{f f}\right)_{i j}= & {\left[q_{i j} /\left(\lambda_{i} \bar{\lambda}_{j} \Delta g(t)^{2}\right)\right] \int_{r=0}^{\Delta g(t)}\left(\exp \left\{\lambda_{i}(\Delta g(t)-r)\right\}-1\right) } \\
& \times\left(\exp \left\{\bar{\lambda}_{j}(\Delta g(t)-r)\right\}-1\right) d r \\
= & \left\{Q_{t}^{s s}+\left(1-W_{i t}-\bar{W}_{j t}\right) q_{i j} \Delta g(t)\right\} /\left(\lambda_{i} \bar{\lambda}_{j} \Delta g(t)^{2}\right) .
\end{aligned}
$$

In addition, under the assumption that $g(t)$ is piecewise linear, $W_{t}$ simplifies to $W_{t}=(\Lambda \Delta g(t))^{-1}\left(T_{t}-I\right)$.

The final set of integrals involve the deterministic component of the continuous-time models, $X(s ; \beta)$. These integrals are evaluated here for a linear time trend in operational time, $X(s ; \beta)=$ $\beta_{0}+\beta_{1} s$, for which the components of $\gamma_{t}$ are

$$
\gamma_{t}=\Delta g(t) W_{t} G^{-1} R \beta_{0}
$$

$$
+\left\{\left(g(t) I+\Lambda^{-1}\right) \Delta g(t) W_{t}-\Lambda^{-1} \Delta g(t) T_{t}\right\} G^{-1} R \beta_{1}
$$

and

$$
\gamma_{t}^{f}=B_{1 t} G^{-1} R \beta_{0}+\left(B_{1 t} g(t)+B_{2 t}\right) G^{-1} R \beta_{1},
$$

where $B_{\mathrm{lt}}=\Lambda^{-1}\left(W_{t}-I\right)$ and $B_{2 t}=\Lambda^{-1}\left[\Lambda^{-1}\left(W_{t}-I\right)+\frac{1}{2} \Delta g(t)-\right.$ $\left.(\Delta g(t)) W_{t}\right]$.

[Received December 1985. Revised August 1987.]

\section{REFERENCES}

Bergstrom, A. R. (ed.) (1976), Statistical Inference in Continuous Time Economic Models, Amsterdam: North-Holland.

(1983), "Gaussian Estimation of Structural Parameters in Higher Order Continuous Time Dynamic Models," Econometrica, 51, 117152.

Burns, A. F., and Mitchell, W. C. (1946), Measuring Business Cycles, New York: National Bureau of Economic Research.

Engle, R. F. (1982), "Autoregressive Conditional Heteroscedasticity With Estimates of the Variance of United Kingdom Inflations," Econometrica, 50, 987-1008.

Friedman, M., and Schwartz, A. J. (1982), Monetary Trends in the United States and the United Kingdom: Their Relation to Income Prices, and Interest Rates, 1867-1975, Chicago: University of Chicago Press.

Harvey, A. C. (1985), "Trends and Cycles in Macroeconomic Time Series," Journal of Business \& Economic Statistics, 3, 216-227.

Harvey, A. C., and Stock, J. H. (1985), "The Estimation of Higher Order Continuous Time Autoregressive Models," Econometric Theory, 1, 97-112.

Jones, R. H. (1981), "Fitting a Continuous Time Autoregression to Discrete Data," in Applied Time Series Analysis II, ed. D. Findley, New York: Academic Press, pp. 651-682.

Kalman, R. E., and Bucy, R. S. (1961), "New Results in Linear Filtering and Prediction Theory," Transactions of the ASME, Ser. D-Journal of Basic Engineering, 83, 95-108.

Neftci, S. N. (1984), "Are Economic Time Series Asymmetric Over the Business Cycle?” Journal of Political Economy, 92, 307-328. (1985), "A Note on the Use of Local Maxima to Predict Turning Points in Related Series," Journal of the American Statistical Association, 80, 553-557.

Nelson, C. R., and Plosser, C. I. (1982), "Trends and Random Walks in Macroeconomic Time Series: Some Evidence and Implications," Journal of Monetary Economics, 10, 139-162.

Phadke, M. S., and Wu, S. M. (1974), "Modeling of Continuous Stochastic Processes From Discrete Observations With Application to 
Sunspots Data," Journal of the American Statistical Association, 69, 325-329.

Phillips, A. W. (1959), "The Estimation of Parameters in Systems of Stochastic Differential Equations," Biometrika, 46, 67-76.

Phillips, P. C. B. (1972), "The Structural Estimation of a Stochastic Differential Equation System," Econometrica, 40, 1021-1041.

- (1974), "The Estimation of Some Continuous Time Models," Econometrica, 42, 803-824.

Robinson, P. M. (1976a), "The Estimation of Linear Differential Equations With Constant Coefficients," Econometrica, 44, 751-764.

(1976b), "Instrumental Variables Estimation of Differential Equations," Econometrica, 44, 765-776. (1977a), "The Construction and Estimation of Continuous Time Models and Discrete Approximations in Econometrics," Journal of Econometrics, 6, 173-198.

(1977b), "Estimation of a Time Series Model From Unequally Spaced Data," Stochastic Processes and Their Applications, 6, 9-24. Stock, J. H. (1983), "Dynamic Economic Models Subject to Time Deformation," unpublished Ph.D dissertation, University of California, Berkeley, Dept. of Economics.

(1987), "Measuring Business Cycle Time," Journal of Political Economy, 95, 1240-1261.

Wymer, C. R. (1972), "Econometric Estimation of Stochastic Differential Equation System," Econometrica, 40, 565-577. 